\title{
TWO-DIMENSIONAL NONCOMMUTATIVE FIELD THEORY ON THE LIGHT CONE
}

\author{
MAHOUTON N. HOUNKONNOU, FORTUNÉ MASSAMBA and JOSEPH \\ BEN GELOUN
}

Communicated by Gerald A. Goldin

Abstract. The two-dimensional noncommutative field theory on the light cone is studied from the usual Moyal product formalism. The Noether currents and the components of the energy-momentum tensor are explicitly computed.

\section{Introduction}

One of the major outstanding problems in Physics is the calculation of observable processes in strongly interacting field theories like QCD and electroweak theory. In particular, it is difficult to calculate, from first principles, the hadronic spectrum, structure functions, fragmentation functions, weak decay amplitudes and nuclear structure. The two most promising attempts to tackle strongly interacting field theories are lattice calculations and light cone field theory. In the 60's, Fubini and Furlan [6] showed that, in a Poincaré invariant theory, calculations may be simpler in an "infinite momentum frame", i.e., in a frame moving with a velocity $v \rightarrow c$ (the light velocity) relative to the centre of mass. Weinberg showed that the singularities for $\gamma=\frac{1}{\sqrt{1-\left(\frac{v}{c}\right)^{2}}} \rightarrow \infty$ cancel in the physical observables [15]. The net effect (apart from a singular scale factor) is to transform to the light cone coordinates

$x^{+}:=\frac{1}{\sqrt{2}}\left(x^{0}+x^{3}\right), \quad x^{-}:=\frac{1}{\sqrt{2}}\left(x^{0}-x^{3}\right), \quad x_{\perp}=\left(x^{1}, x^{2}\right)$ unaffected

with $x^{+}$regarded as the (light cone) time and $x^{-}, x^{1}$ and $x^{2}$ regarded as spatial coordinates. This interpretation is crucial as the Hamiltonian formalism does not treat space and time in a symmetric way.

Recently, the investigation of the gauge field theories in a noncommutative spacetime has become of increasing interest [1-6], [8-10], [12-15]. 
In this paper, we investigate the two-dimensional noncommutative field theory on the light cone from the Moyal product formalism. We deduce, from the variational principle, the Noether currents and the components of the energy-momentum tensor.

The paper is organized as follows. In Section 2, we provide the usual commutative gauge theories on the light cone. In Section 3, the theoretical framework of the noncommutative spacetime on the light cone is given; the Moyal product is presented. The noncommutative $\mathrm{U}(1)$ covariant derivative and gauge action are investigated as well as the equations of motion and the gauge invariance of the theory. Furthermore, the Noether Theorem is introduced and the corresponding currents are explicitly computed. In Section 4, the components of the energy momentum tensor are calculated with respect to the $\star$-product.

\section{Two-Dimensional Commutative Gauge Theory on the Light Cone}

Consider the following Lagrangian density [11]

$$
L=\bar{\chi}\left(\mathrm{i} \partial_{\mu}+g A_{\mu}\right) \gamma^{\mu} \chi-m \bar{\chi} \chi-\frac{1}{4} F_{\mu \nu} F^{\mu \nu} .
$$

The electromagnetic field is described by the vector potential $A_{\mu}(\mu=0,1)$ which in turn determines the field strength tensor

$$
F_{\mu \nu}=\partial_{\mu} A_{\nu}-\partial_{\nu} A_{\mu}
$$

The matter field is described by a two component spinor

$$
\chi=\left(\begin{array}{c}
\phi \\
\psi
\end{array}\right)
$$

with $\phi$ and $\psi$ being Grassmann variables. The $\gamma^{\mu}$ are $2 \times 2$ Dirac matrices satisfying the Clifford algebra relations

$$
\left\{\gamma^{\mu}, \gamma^{\nu}\right\}=2 g^{\mu \nu}, \quad g^{\mu \nu}=\left(\begin{array}{cc}
1 & 0 \\
0 & -1
\end{array}\right) .
$$

In addition, we set

$$
\gamma^{5}=\gamma^{0} \gamma^{1}=\left(\begin{array}{cc}
1 & 0 \\
0 & -1
\end{array}\right)
$$


To simplify further the Lagrangian density, new coordinates are introduced

$$
\begin{array}{lll}
x^{+}=\frac{1}{\sqrt{2}}\left(x^{0}+x^{1}\right), & \partial_{+}=\frac{1}{\sqrt{2}}\left(\partial_{0}+\partial_{1}\right) \\
x^{-}=\frac{1}{\sqrt{2}}\left(x^{0}-x^{1}\right), & \partial_{-}=\frac{1}{\sqrt{2}}\left(\partial_{0}-\partial_{1}\right)
\end{array}
$$

with a new combination of the gauge fields:

$$
A_{ \pm}=\frac{1}{\sqrt{2}}\left(A_{0} \pm A_{1}\right) .
$$

The metric in these two-dimensional light cone coordinates is

$$
g_{--}=g_{++}=0, \quad g_{-+}=g_{+-}=1, \quad g^{--}=g^{++}=0, \quad g^{-+}=g^{+-}=1 .
$$

The commutative Lagrangian (1) thus becomes

$$
\begin{aligned}
L= & \sqrt{2} \phi^{\dagger}\left(\mathrm{i} \partial_{+}+g A_{+}\right) \phi+\sqrt{2} \psi^{\dagger}\left(\mathrm{i} \partial_{-}+g A_{-}\right) \psi \\
& -m\left(\phi^{\dagger} \psi+\psi^{\dagger} \phi\right)+\frac{1}{2}\left(\partial_{-} A_{+}-\partial_{+} A_{-}\right)^{2} .
\end{aligned}
$$

\section{Noncommutative Gauge Theory on the Light Cone}

The noncommutative geometric spaces are usually described by a $\star$-algebra. In general, this algebra is not commutative. The usual product of functions is therefore replaced by the $\star$-product, which is characteristic for the noncommutative geometry and is given by

$$
(f \star g)(x)=\exp \left(\frac{1}{2} \mathrm{i} \theta^{n m} \partial_{x^{n}} \partial_{y^{m}}\right) f(x) g(y)_{\mid x=y}
$$

where $f$ and $g$ are arbitrary smooth functions, and $\theta^{n m}$ is a real constant antisymmetric tensor. The Moyal bracket of two functions is $[f, g]_{\star}=f \star g-g \star f$. If we choose $f$ and $g$ to be the light cone coordinates, we obtain $\left[x^{-}, x^{+}\right]_{\star}=\mathrm{i} \theta^{-+}$, where $\theta^{-+}=\theta \varepsilon^{-+}, \varepsilon^{-+}=-\varepsilon^{+-}=1, \theta=$ constant.

\subsection{Noncommutative U(1) Gauge Action}

Define the noncommutative $\mathrm{U}(1)$ covariant derivative as

$$
D_{ \pm}(\cdot)=\partial_{ \pm}(\cdot)-\mathrm{i} g A_{ \pm} \star(\cdot)
$$


with the bracket given by

$$
\left[D_{-}, D_{+}\right]_{\star}(\cdot)=-\mathrm{i} g F_{-+}(\cdot)
$$

where $F_{-+}=\partial_{-} A_{+}-\partial_{+} A_{-}-\mathrm{i} g\left[A_{-}, A_{+}\right]_{\star}$. Equation (10) is called the Ricci identity and the noncommutative field obtained from this identity can be identified as the noncommutative curvature tensor.

The noncommutative gauge action can be written as

$$
\begin{aligned}
S= & \int \mathrm{d}^{2} x\left\{\mathrm{i} \sqrt{2} \phi^{\dagger} \star D_{+} \phi+\mathrm{i} \sqrt{2} \psi^{\dagger} \star D_{-} \psi-m\left(\phi^{\dagger} \star \psi+\psi^{\dagger} \star \phi\right)\right\} \\
& +\frac{1}{2} \int \mathrm{d}^{2} x F_{-+} \star F_{-+} .
\end{aligned}
$$

\subsection{Equations of Motion}

Equations of motion are derived through the stationary action principle. This principle states that the quantities of the system follow an extremum path in phase space. From the variation of the action and the properties of the $\star$-product, and setting the surface terms $\partial_{+}\left(\phi^{\dagger} \star \delta \phi\right), \partial_{-}\left(\psi^{\dagger} \star \delta \psi\right), \partial_{-}\left(\delta A_{+} \star F_{-+}\right)$and $\partial_{+}\left(\delta A_{-} \star F_{-+}\right)$to zero, we deduce the following equations of motion

$$
\begin{aligned}
& \mathrm{i} \sqrt{2} D_{+} \phi-m \psi=0, \quad \mathrm{i} \sqrt{2} D_{-} \psi-m \phi=0 \\
& -\mathrm{i} \sqrt{2} \bar{D}_{+} \phi^{\dagger}-m \psi^{\dagger}=0, \quad-\mathrm{i} \sqrt{2} \bar{D}_{-} \psi^{\dagger}-m \phi^{\dagger}=0 \\
& -g \sqrt{2} \phi \star \phi^{\dagger}-\partial_{-} F_{-+}+\mathrm{i} g\left[A_{-}, F_{-+}\right]_{\star}=0 \\
& -g \sqrt{2} \psi \star \psi^{\dagger}+\partial_{+} F_{-+}-\mathrm{i} g\left[A_{+}, F_{-+}\right]_{\star}=0 .
\end{aligned}
$$

In this case (14) and (15) are the noncommutative counterparts of Maxwell equations on the light cone. With $\mathcal{D}_{ \pm} \cdot=\partial_{ \pm} \cdot-\mathrm{i} g\left[A_{ \pm}, \cdot\right]$, they can be written as

$$
\mathcal{D}_{-} F_{-+}=-g \sqrt{2} \phi \star \phi^{\dagger}, \quad \mathcal{D}_{+} F_{-+}=g \sqrt{2} \psi \star \psi^{\dagger}
$$

which define the noncommutative Gauss-Law in the light cone representation.

\subsection{Gauge Invariance of the Theory}

Let $\lambda(x)$ be a function on the spacetime of the light cone, $U(x)$ - a noncommutative $\mathrm{U}(1)$ gauge transformation defined by $U(x)=\mathrm{e}_{\star}^{\mathrm{i} \lambda}=1+\mathrm{i} \lambda-\frac{1}{2} \lambda \star$ $\lambda-\frac{\mathrm{i}}{3 !} \lambda \star \lambda \star \lambda+\cdots$ Using the definition of the $\star$-product, we can show that 
$U(x) \star U^{-1}(x)=U^{-1}(x) \star U(x)=1$. Under the noncommutative $\mathrm{U}(1)$ gauge group, the fermionic part is transformed as

$$
(\phi, \psi) \rightarrow(U \star \phi, U \star \psi), \quad\left(\phi^{\dagger}, \psi^{\dagger}\right) \rightarrow\left(\phi^{\dagger} \star U^{-1}, \psi^{\dagger} \star U^{-1}\right)
$$

and the gauge part is transformed as, with $x=\left(x^{+}, x^{-}\right)$

$$
A_{ \pm} \rightarrow A_{ \pm}^{\prime}=\frac{\mathrm{i}}{g} U(x) \star D_{ \pm} U^{-1}(x), F_{-+} \rightarrow F_{-+}^{\prime}=U(x) \star F_{-+} \star U^{-1}(x) .
$$

Proposition 1. The infinitesimal gauge transformation is given, for the fermionic and gauge parts, respectively, by

$$
\begin{aligned}
(\delta \phi, \delta \psi) & =(\mathrm{i} \lambda \star \phi, \mathrm{i} \lambda \star \psi),\left(\delta \phi^{\dagger}, \delta \psi^{\dagger}\right)=\left(-\mathrm{i} \phi^{\dagger} \star \lambda,-\mathrm{i} \psi^{\dagger} \star \lambda\right) \\
\delta A_{ \pm} & =\frac{1}{g} \mathcal{D}_{ \pm} \lambda .
\end{aligned}
$$

Under the noncommutative $\mathrm{U}(1)$ gauge transformations (17) and (18), the noncommutative action is gauge invariant.

\subsection{Noether Currents}

The Noether theorem allows us to deduce the current density via the infinitesimal variation of the action $S$. Using the equations of motion (14) and (15), the Noether currents corresponding, respectively, to the fermionic and gauge parts, with respect to the axis $x^{+}$and $x^{-}$, are given by the expressions

$$
\left(J^{+}, J^{-}\right)=\left(\phi \star \phi^{\dagger}, \psi \star \psi^{\dagger}\right), \quad\left(J_{e}^{+}, J_{e}^{-}\right)=\left(-g \phi \star \phi^{\dagger}, g \psi \star \psi^{\dagger}\right) .
$$

$J_{e}^{ \pm}$can be also called the noncommutative electromagnetic currents with respect to the axis $x^{ \pm}$of the light cone.

The fermionic charge is obtained as

$$
Q=\int \mathrm{d} x^{+} J^{+}=\int \mathrm{d} x^{+} \phi \star \phi^{\dagger}
$$

while the axial current $J_{\gamma_{5}}^{n}$ under axial symmetry $\mathrm{e}_{\star}^{\mathrm{i} \lambda \gamma_{5}}$ is

$$
J_{5}^{+}=-\phi \star \phi^{\dagger}, \quad J_{5}^{-}=\psi \star \psi^{\dagger} .
$$

With expressions (21), the Noether currents are related by

$$
\left(J_{e}^{+}, J_{e}^{-}\right)=\left(g J^{+},-g J^{-}\right) .
$$


Proposition 2. Under the equations (14) and (15) we have

$$
\mathcal{D}_{+} J^{+}+\mathcal{D}_{-} J^{-}=0 .
$$

This is the light cone version of the Ward identity [2], which means that the Noether currents are (due to the equations of motion and the Bianchi identity) covariantly conserved.

Proposition 3. Under the equations (14) and (15) we have also

$$
\partial_{+} J^{-}+\partial_{-} J^{+}=\frac{\mathrm{i}}{\sqrt{2}}\left(\partial_{+}\left[A_{-}, F_{-+}\right]_{\star}-\partial_{-}\left[A_{+}, F_{-+}\right]_{\star}\right) .
$$

The Noether currents are not locally conserved, but the integral over the noncommutative light cone coordinates of these divergence vanishes.

\section{Energy-Momentum Tensor in the Light Cone Representation}

In this Section, we compute the energy-momentum tensor using the noncommutative Lagrangian density whose terms depend on the derivatives through the definition of the $\star$-product. The noncommutative Lagrangian density $\mathcal{L}$ is written as commutative terms by perturbing the initial Lagrangian density by an interaction Lagrangian. This is obtained by the expansion of the Lagrangian using the $\star$-product definition (8) in the first $\theta$-order. From the conjugate momentum with respect to the fermionic and gauge parts of the Lagrangian density $\mathcal{L}$, the calculation of the components of the energy-momentum tensors becomes easier. We obtain in a straightforward way

$$
\begin{aligned}
T^{++}= & -\mathrm{i}\left(\sqrt{2} \phi^{\dagger}+\frac{1}{2} \theta \partial_{-}\left(g \sqrt{2}\left(\phi^{\dagger} \star A_{+}\right)-m \psi^{\dagger}\right)\right) \star \partial_{+} \phi \\
& -\frac{\mathrm{i}}{2} \theta \partial_{-}\left(g \sqrt{2}\left(\psi^{\dagger} \star A_{-}\right)-m \phi^{\dagger}\right) \star \partial_{+} \psi \\
& +\frac{1}{2} g \theta\left(\mathrm{i} \sqrt{2}\left(\partial_{-} \phi^{\dagger} \star \phi-\phi^{\dagger} \star \partial_{-} \phi\right)+\left\{\partial_{-} A_{-}, F_{-+}\right\}_{\star}\right) \star \partial_{+} A_{+} \\
& +\frac{1}{2}\left(\mathrm{i} g \sqrt{2} \theta\left(\partial_{-} \psi^{\dagger} \star \psi-\psi^{\dagger} \star \partial_{-} \psi\right)-2 F_{-+}-g \theta\left\{\partial_{-} A_{+}, F_{-+}\right\}_{\star}\right) \\
& \star \partial_{+} A_{-}
\end{aligned}
$$

which is the Hamiltonian density; the brackets $\{(\cdot),(\cdot)\}$ are defined as, for any $f$ and $g,\{f, g\}_{\star}=f \star g+g \star f$. The component $T^{+-}$of the energy-momentum 
tensor is respectively

$$
\begin{aligned}
T^{+-}= & -\mathrm{i}\left(\sqrt{2} \phi^{\dagger}+\frac{1}{2} \theta \partial_{-}\left(g \sqrt{2}\left(\phi^{\dagger} \star A_{+}\right)-m \psi^{\dagger}\right)\right) \star \partial_{-} \phi \\
& -\frac{\mathrm{i}}{2} \theta \partial_{-}\left(g \sqrt{2}\left(\psi^{\dagger} \star A_{-}\right)-m \phi^{\dagger}\right) \star \partial_{-} \psi \\
& +\frac{1}{2} g \theta\left(\mathrm{i} \sqrt{2}\left(\partial_{-} \phi^{\dagger} \star \phi-\phi^{\dagger} \star \partial_{-} \phi\right)+\left\{\partial_{-} A_{-}, F_{-+}\right\}_{\star}\right) \star \partial_{-} A_{+} \\
& +\frac{1}{2}\left(\mathrm{i} g \sqrt{2} \theta\left(\partial_{-} \psi^{\dagger} \star \psi-\psi^{\dagger} \star \partial_{-} \psi\right)-2 T_{-+}^{F}-g \theta\left\{\partial_{-} A_{+}, F_{-+}\right\}_{\star}\right) \\
& \star \partial_{-} A_{-}-\mathcal{L} .
\end{aligned}
$$

The other components of the energy-momentun tensor, namely, $T^{-+}$and $T^{--}$ can be obtained in a similar way [10]. We find that the energy-momentum tensor is neither locally nor covariantly conserved, i.e., for $n, m=+,-$

$$
\partial_{n} T^{n m} \neq 0, \quad D_{n} T^{n m} \neq 0
$$

This result (29) is well in a good agreement with the work of Grimstrup et al [8] who have studied the particular case of the gauge action.

\section{Acknowledgements}

One of the authors, M. N. H., thanks Professor R. J. Szabo (School of Mathematical and Computer Sciences, Heriot-Watt University, Edinburg, United Kingdom) for helpful discussions.

\section{References}

[1] Aharony O., Gomis J. and Mehen T., On Theories with Light-Like Noncommutativity, JHEP 0009 (2000) 023.

[2] Ardalan F. and Sadooghi N., Anomaly and Nonplanar Diagrams in Noncommutative Gauge Theories, Int. J. of Mod. Phys. A 17 (2002) 123-144.

[3] Caroll M., Harvey A., Kostelecky A., Lane D. and Okamoto T., Noncommutative Field Theory and Lorentz Violation, Phys. Rev. Lett. 87 (2001) 141601.

[4] Connes A., Douglas M. and Schwarz A., Noncommutative Geometry and Matrix Theory: Compactification on Tori, JHEP 02 (1998) 003. 
[5] Connes A. and Rieffel M., Yang Mills for Noncommutative Two Tori, Contemp. Math. Oper. Algebra. Math. Phys. 62 (1987) 237-266.

[6] Fubini S. and Furlan G., Renormalization Effects for Partially Conserved Currents, Physics 1 (1965) 229-247.

[7] Geloun J., Hounkonnou N. and Massamba F., On Some Algebraic Computations in Noncommutative Field Theory, ICMPA Preprint MPA/2005/09.

[8] Grimstrup J., Kloiböck B., Popp L., Putz V., Schweda M. and Wickenhauser M., The Energy-Momentum Tensor in Noncommutative Gauge Field Models, Int. J. Mod. Phys. A 19 (2004) 5615-5624.

[9] Hayakawa M., Perturbative Analysis on Infra-red and Ultraviolet Aspects of Noncommutative QED on $\mathbb{R}^{4}$, Phys Lett. B 478 (2000) 394-400.

[10] Hounkonnou N., Massamba F. and Geloun J., Light Cone Gauge Field Theory in a Noncommutative 2D Spacetime, ICMPA Preprint MPA/2005/08.

[11] Lenz F., Thies M., Levit S. and Yazaki K., Hamiltonian Formulation of TwoDimensional Gauge Theories on the Light Cone, Ann. Phys. 208 (1991) $1-89$.

[12] Madore J., An Introduction to Noncommutative Geometry and its Physical Applications, Second Edition, Cambridge Univ. Press, Cambridge, 1999.

[13] Micu A. and Sheikh-Jabbari M., Noncommutative $\Phi^{4}$ Theory at Two Loops, JHEP 0101 (2001) 025.

[14] Seiberg N. and Witten E., String Theory and Noncommutative Geometry, JHEP 9909 (1999) 032.

[15] Weinberg S., Dynamics at Finite Momentum, Phys. Rev. 150 (1966) 13131318.

Mahouton Norbert Hounkonnou

International Chair in Mathematical

Physics and Applications (ICMPA)

072 B.P.: 50 Cotonou

REPUBLIC of BENIN

E-mail address: norbert_hounkonnou@cipma. net

Fortuné Massamba

International Chair in Mathematical

Physics and Applications (ICMPA)

072 B.P.: 50 Cotonou

REPUBLIC of BENIN

E-mail address: massfort@yahoo.fr 


\author{
Joseph Ben Geloun \\ International Chair in Mathematical \\ Physics and Applications (ICMPA) \\ 072 B.P.: 50 Cotonou \\ REPUBLIC of BENIN
}

E-mail address: jobengeloun@yahoo.fr 